\title{
CONTACT LENSES IN TREATMENT OF KERATOCONUS*
}

\author{
BY \\ FREDERICK RIDLEY \\ London
}

THE increasing recognition of the importance of contact lenses in the treatment of keratoconus has led to a quite exceptional concentration of these cases in the Contact Lens Department at the Royal Westminster branch of the Moorfields, Westminster and Central Eye Hospital. It is possible that the opportunity to analyse in detail such a large series of keratoconus cases has not previously occurred, and it is proposed to present the general picture of the disease as seen in these cases and to draw certain conclusions, before proceeding to consider in greater detail the treatment of keratoconus using contact lenses.

\section{Material}

Between April, 1951, and September, 1955, rather more than 2,000 patients were seen at the Contact Lens Department. Of these 2,000 patients, 92 were sent with a diagnosis of keratoconus. They came from all over the British Isles and indeed several from abroad. Most of the cases were sent because the affected eye or eyes were not adequately corrected by spectacles. Many were sent hoping that contact lenses would prove an alternative to surgery. To this extent the material.is selected, and it is probable that it is weighted fairly heavily in the direction of severe cases, especially in the older age groups. It is unlikely, however, to be weighted in any way as regards sex, right or left eye, age, or other characteristic, except that we may have seen relatively few monocular cases. All these patients have had a routine medical examination. No special clinical inquiry directed to the problem of keratoconus has been undertaken.

\section{Clinical Findings}

Secondary Diagnoses. - The following secondary diagnoses were recorded: interstitial keratitis with cataract and nebulae (two cases), squint (one), iridectomy (one), myopic fundus degeneration (one).

In one case cataracts had been removed in both eyes, and one instance of unilateral astigmatism of $12 \mathrm{D}$ may not have been a true case of keratoconus. Where these findings would vitiate the investigation, individual cases have been excluded.

-Receivod for publication January 17, 1956. 
Allergy. $-15 \cdot 2$ per cent. of these 92 cases gave a history of allergy, including hay fever, 7.6 per cent.; asthma, 4.3 per cent.; allergic dermatitis, $3 \cdot 3$ per cent.

The general rate, excluding keratoconus, for 2,000 cases is $4 \cdot 2$ per cent., so that keratoconus patients show an incidence of allergy 3.65 times gi eater than the average of all cases seen in the department. These cases of allergy were distributed evenly as to age and sex.

Lenticonus.-Residual astigmatism when a contact lens is worn may be presumed to be lenticular. In this series, ten cases showed residual astigmatism exceeding $0.75 \mathrm{D}$. Both eyes were affected in five cases and one eye in five cases. Of the fifteen eyes thus affected, six showed 1D, five 2D, three 3D, and one 4D of astigmatism. It is fairly certain that many cases of symmetrical lenticonus, not revealed by this method of investigation, also exist. Lenticonus complicating keratoconus does not seem to be related to the severity of the disease.

Keratoconus Posticus.-This was recorded in six cases.

General Observations.-Scrutiny of each of the 92 cases shows that almost invariably the eye with the greater total of refractive error has the worse vision and was the first eye to be affected. For statistical purposes this assumption has been regarded as true throughout the investigation.

There were 42 males and fifty females in this series, evenly distributed in all age groups ranging from 8 to 70 years of age. Right and left eyes were similarly affected as between males and females at all ages; the evidence does not suggest that the right or left eye is more frequently the first eye to be affected in either sex.

As a rule, the active phase of the disease is over by the time the patient has attained 27 years of age: of the cases over 27 years of age, 12.75 per cent. still had 6/6 vision in one eye with spectacles. The true figure for unilateral cases is certainly higher than this, since it is to be presumed that many people having a visual acuity of $6 / 6$ in one eye will not seek relief, or will not have been referred to the department.

In 59 cases, it was possible to record the age at which the disease first became apparent. Fig. 1 shows that the disease rarely begins outside the ages 10 to 25 , and that 67.8 per cent. of all cases are first observed between the ages of 10 and 20 , with a peak at $16 \cdot 1$ years.

Course of the Disease.-The first eye to be affected follows a more severe course than the second eye in most cases. Accordingly the first and second eye affected have been treated as two distinct groups. Fig. 2 shows how the mean visual acuity in the better and worse eyes changes from year to year up to 8 years from the date when the case is first diagnosed. 41 cases were available for this investigation. The following conclusions were reached:

(1) The course of the disease is not influenced by the age when it is first observed. 


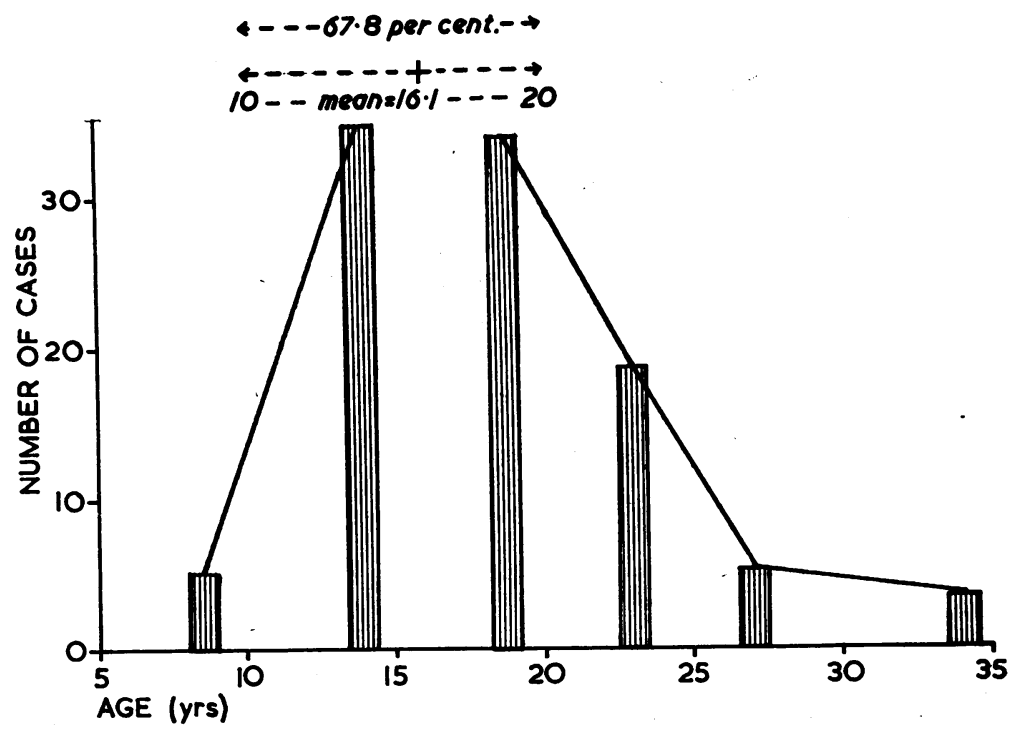

FIG. 1.-Age at which disease was first observed, in 5-year age groups.

FIG. 2.-Average changes in visual acuity in better and worse eyes from time of diagnosis for periods up to 8 yrs. Number of patients observed shown in italics for each period of time.

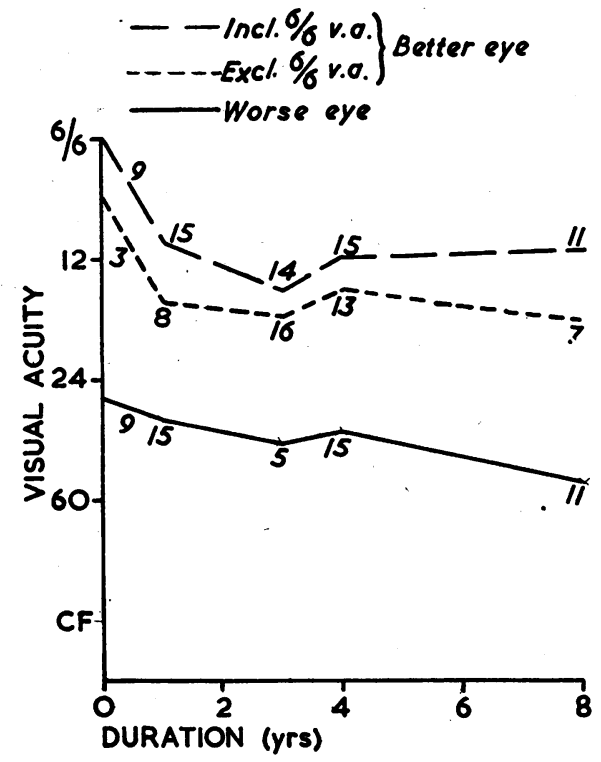

(2) The first eye to be affected is often in the fully-developed stage of the disease when first seen and deteriorates little subsequently.

(3) The second eye affected shows a different picture. The mean visual acuity of this group drops from $6 / 6$ to between $6 / 12$ and $6 / 18$ in the first 2 or 3 years of observation. It then remains fairly steady for the rest of the 8 years as compared with the 'mean figure of $6 / 36$ for the first eye affected. The curves for the second eye are parallel whether we exclude or include eyes having $6 / 6$ vision with spectacles, and it is probable that this is indeed the course of the disease in the second eye. 


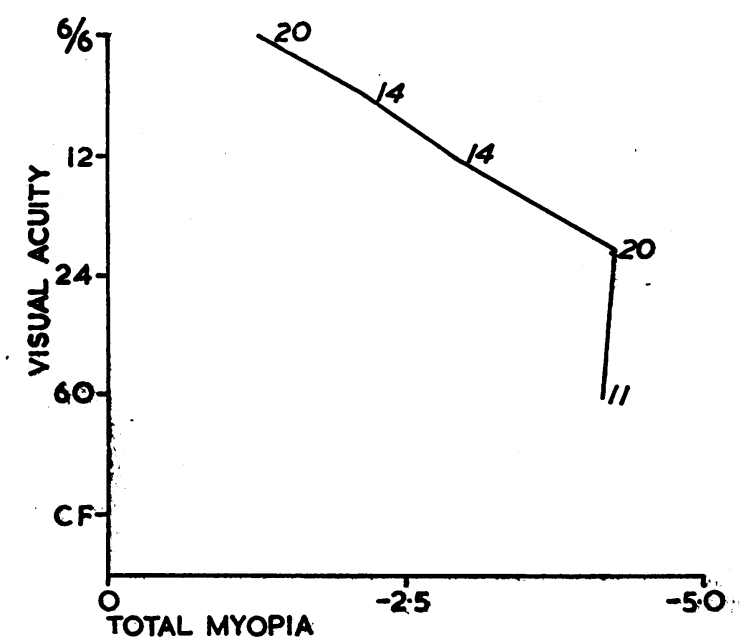

Fig. 3.-Average correctable myopia in better eye at time of diagnosis.

Fig. 3 shows that, on the average, the better eye already has about $-1 \cdot 5 \mathrm{D}$ myopia correctable to $6 / 6$ when the case comes under observation. The distribution of refractive error at each level of visual acuity, which we shall consider later (Fig. 5), suggests that the great majority of these cases are alr eady suffering from the disease and have deteriorated from a probable emmetropia. The disease is one of " giving way " of the central area of the cornea. In most cases this is at first regular in character and may be seen either as increasing relative myopia or increasing astigmatism or both. This may progress to as much as 4 dioptres in all, but at any point irregular stretching may supervene with deterioration of visual acuity with spectacles. It is unusual for a cornea to stretch in a regular manner so as to give more than 4 dioptres of total relative myopia before the stretching becomes irregular and clinical keratoconus supervenes.

The degree of astigmatism, as such, seems to have no direct relation to the visual acuity. Both spherical and astigmatic stretching may remain regular and correctable by spectacles, in exceptional cases, up to quite high figures. The presence of undoubted keratoconus in the fellow eye may be held to establish the diagnosis of keratoconus in such eyes as show these exceptional degrees of refractive error. This group includes Amsler's " keratoconus fruste".

In any case in which deterioration of vision not correctable by spectacles is observed, it may be possible that we are dealing with an anomalous keratoconus. I have had under observation for some years a woman now 52 , who between 43 and 48 years of age, being a myope -10D with pronounced myopic fundus changes involving the macular area, suffered a progressive deterioration of vision from $6 / 6$ to $6 / 18$ with spectacles. Her cornea showed a slight degree of keratoconus and her vision improved to $6 / 6$ with contact lenses. Such cases may be numerous if looked for. 
Distribution of Total Refractive Error.-Hitherto we have not obtained a clear clinical picture of the initial state of refraction in keratoconus. To ascertain this, those cases have been extracted in which a reliable refraction could be done and in which visual acuity was not worse than 6/36 with spectacles. Figs 4 and 5 show that 52 per cent. of eyes in patients with keratoconus have between +2 and $-2 \mathrm{D}$ of total error, and that 78 per cent. have between +2 and $-5 \mathrm{D}$. They are distributed roughly on a binomial curve similar to that for the general population (Fig. 4). This is true of all visual acuity groups $6 / 6$ to $6 / 36$ (Fig. 5). We may conclude that keratoconus attacks a population which is not selected as to initial refraction and centres upon the condition of emmetropia.

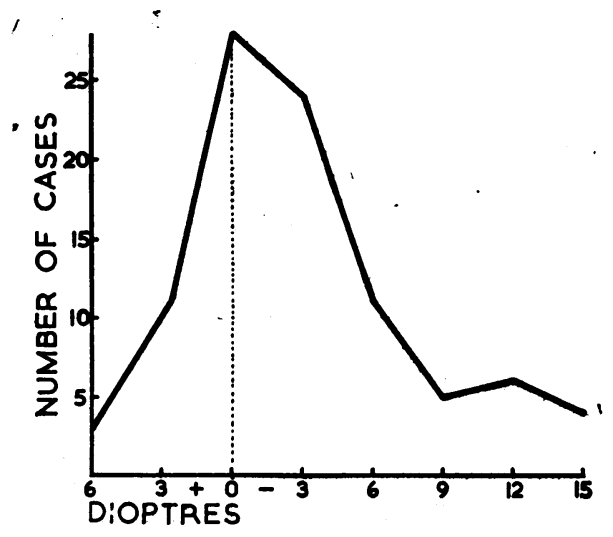

Fig. 4.-Results of refraction, percentage error in 92 cases.

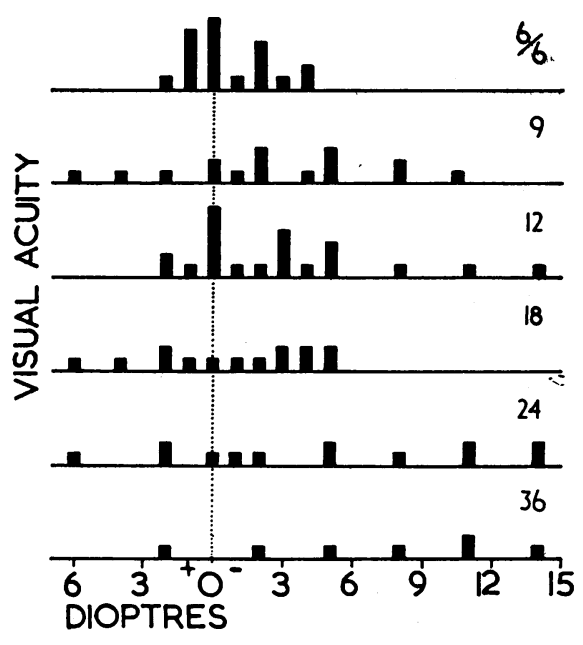

Fig. 5.-Results of refraction, degree of error related to degree of visual acuity.

This suggests that fellow eyes, still having $6 / 6$ vision when the case is first observed (but as we saw previously, having an average total myopia of $-1 \cdot 5 \mathrm{D})$, are in most cases already affected by the disease.

Age at Onset.-The recognition that keratoconus affects an unselected body of people as regards initial refraction enables us to say that if one eye is already affected by keratoconus any increase in relative total myopia (and this may often mean a change from say +2 to $+1.5 \mathrm{D}$ ) is presumptive evidence that the second eye is already affected by the disease.

Taken in conjunction with Fig. 3, the slope of the graphs in Fig. 2 for the second eye affected suggests that, on the average, the relative myopia progresses at about $1 \mathrm{D}$ per year. This would place the occurrence of the true onset of the disease about 18 months before the visual acuity with spectacles begins to deteriorate in an average case.

Identification of Active Disease. - In the present state of knowledge we cannot as a rule diagnose the first eye clinically until its visual acuity with spectacles begins to fall but we can diagnose the condition in the second eye very early 
indeed if we keep a close watch for a change of total refraction almost always towards myopia. It is here that we may study the disease in its early active phase and here too we may hope to ascertain its aetiology and apply treatment successfully. This may well mean frequent, perhaps 3-monthly, refraction under a mydriatic, but in this way we may recognize the condition some 18 months earlier than is otherwise possible.

\section{Fitting Contact Lenses}

Selection of Cases.-Of the 92 cases seen, only nine were found unsuitable for contact lenses. Of the 83 cases accepted for fitting, 56 have been completed and a final result obtained by September, 1955. Of these 56 cases, 34-have had their lenses more than 12 months.

Without exception, any keratoconus in which there is a red reflex should be tried out with contact lenses. Alternatively, any patient who can count fingers at $1 \mathrm{ft}$. with spectacles should give them a trial. There is some evidence that the regular wearing of contact lenses arrests, or at least greatly reduces, the rate of progress of the disease. Perhaps even with 6/6 vision in one eye both eyes should be fitted and contact lenses worn up to the age of 30 when the disease seems almost invariably to have burnt itself out, unless it is feasible to keep the unaffected eye under constant observation.

Allergy and a light-sensitive skin tend to reduce the wearing time and may give rise to periods when the contact lenses cannot be worn. All eyes that are not hopeless should be fitted, and in this I include extreme conus and active ulceration. However extreme the conus, it is almost always possible to fit a contact lens, albeit with much technical difficulty, and in our experience ulcerating cases have healed under a lens and the eye has become comfortable.

Technical Details.-Always mould; always avoid apical touch; in severe cases seek to fit a lens directly off the mould and so avoid dead spaces under the lens and secure a stable fit. In severe conus, orthodox lenses tend to be a very sloppy fit and it is impossible to avoid corneal touch with consequent discomfort. This approach often means that no true optical surface can be worked on the back of the lens, but even so it is possible as a rule to secure a visual acuity of $6 / 9$, and even $6 / 5$ has been achieved. This technique necessitates an almost perfect mould and very careful processing since we are seeking to copy precisely both the form and surface of the cone of the eye. No fitting can be done over this area at all; indeed by this method we try to avoid fitting altogether. In all cases construct the contact lenses with an optimum front contour to secure comfort and symmetry in the two eyes.

Type of Lens.-In severe cases fenestration may be impossible because the apex of the cone lies off, usually below, the optical axis and the bubble would lie in the line of sight. We have to rely in these cases on a lens with minimal apical clearance but provided with a drainage channel or a loose area to facilitate fluid exchange and prevent the development of a negative pressure under the lens, so that veiling will not occur. No lens should be passed 
which " sticks" in use. The ideal lens in many cases of keratoconus is one that is a true glove fit overall.

Fitting Time and Adaptability.-Keratoconus cases are fitted with much the same facility as other types of case and learn to use and wear their lenses equally well and quickly.

Special Problems.- In rare cases of keratoconus grey or partially translucent areas cause optical distortion within the pupil and give rise to blurring of the retinal image even with a contact lens, although there is some clear cornea present; in one case optical iridectomy had been performed in addition. In such cases an opaque contact lens simulating an artificial eye having an optically worked pupillary area may be constructed, in which the clear area of cornea is opposed to the pupil of the contact lens and the grey areas are masked.

\section{Clinical Results}

Immediate Results.-In no other type of case save clear but distorted penetrating corneal grafts are the visual results obtained by contact lenses so dramatic. In each column in Fig. 6 the light shading represents the number of eyes in the present series with a certain degree of visual acuity (wearing spectacles). The dark shading represents the visual acuity attained by each eye wearing a contact lens as opposed to spectacles. The few eyes not improved carry no dark shading above the light column. If an eye with keratoconus can count fingers at $1 \mathrm{ft}$. with spectacles it is very possible that contact lenses will restore useful and even normal vision. Eyes that cannot count fingers at $1 \mathrm{ft}$. are rarely improved.

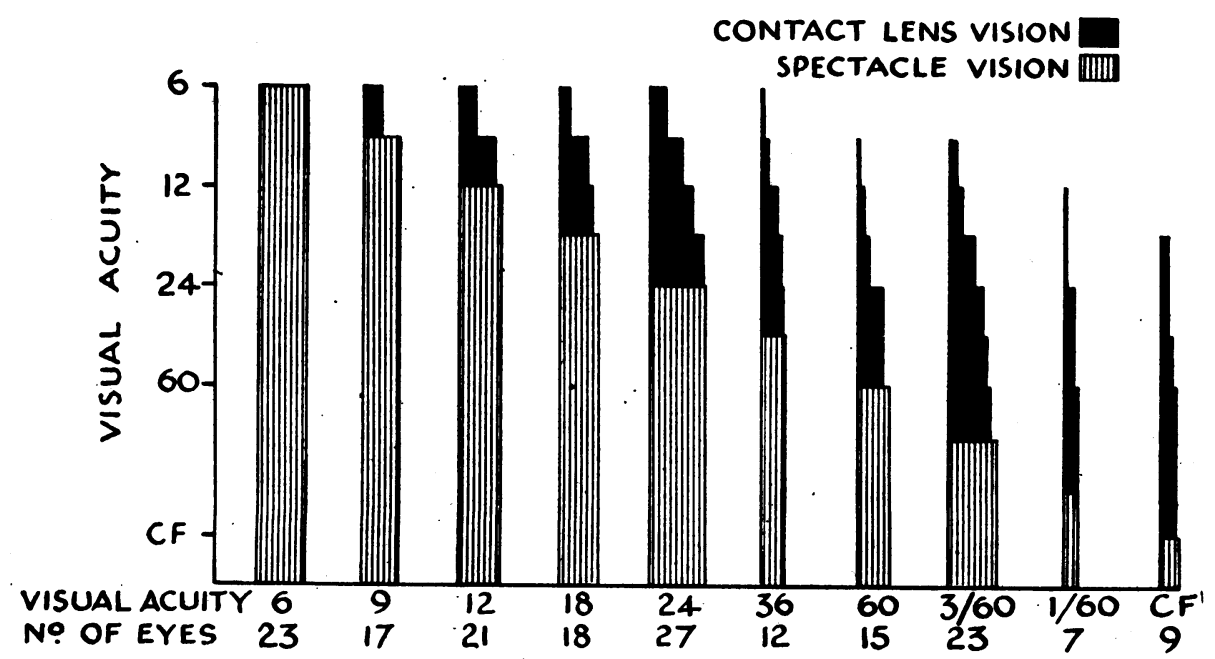

Fig. 6.-Degree of visual improvement with contact lenses as compared with spectacles. 
We may draw general conclusions from Fig 6 by considering the keratoconus patients as a whole. To do this we allot "visual units", ten for 6/6, nine for 6/9, down to one.for counting fingers at $1 \mathrm{ft}$ (Ridley, 1954), and so calculate the number of units at each visual acuity level enjoyed by this group of patients: The right-hand section of Fig. 7 represents the total visual capacity of this group wearing spectacles, distributed at various degrees of acuity. The left-hand section represents the visual capacity of the same set of patients wearing contact lenses. Here not only is the total vision enjoyed greatly increased, but it is concentrated in the higher degrees of visual acuity. Keratoconus patients fitted with contact lenses not only enjoy this improvement in vision, but they are at the same time rendered iseikonic and fully binocular with a full visual field, and the more severe cases are made much more comfortable physically.
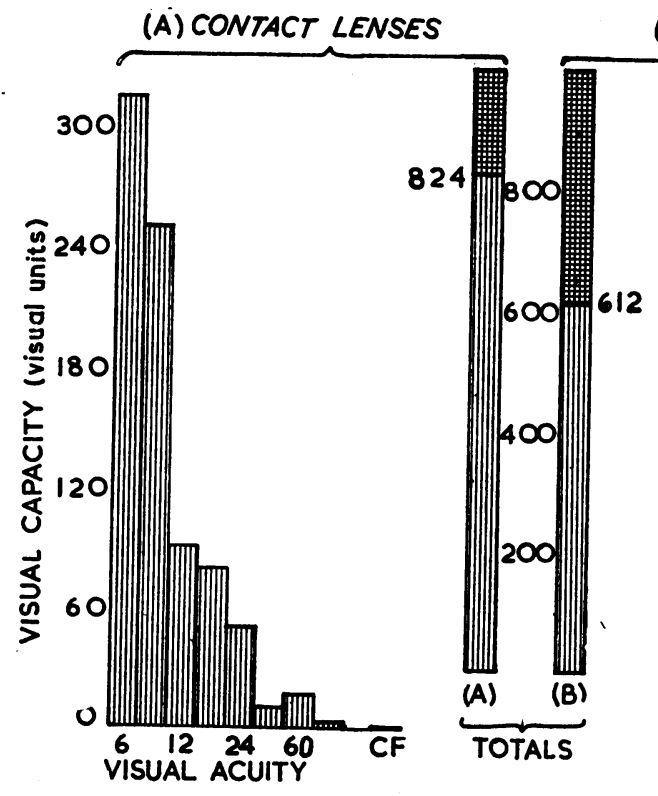

(B) SPECTACLES

Fig. 7.-Visual capacity (calculated in visual units) in keratoconus patients at various levels of visual acuity: (A) using contact lenses; (B) using spectacles. Percentage of 172 eyes.

Long-Term Results. - The effect is probably permanent in most cases. Since the contact lens abolishes the cornea optically, any increase in the cone will show only when corneal touch occurs and the lens has to be hollowed out to accommodate it. This procedure has so far been necessary in only one case in this series.

Wearing Time.-A patient is described as an " all-day wearer" if he has no enforced rest periods in an arbitrary "wearing time" of 16 hours daily. This length of time represents the normal full day and is greater than the 
number of hours that can be achieved by a "discontinuous wearer", who wears his lenses for $x$ periods of $y$ hours a day and has to take one or more enforced rest periods which may vary from $15 \mathrm{~min}$. to one hour. A standard wearing time (S.W.T.) for any case is arrived at by taking the total number of hours a discontinuous wearer wears his lenses in an average day or 16 hours for an all-day wearer. The follow-up of all the cases in the department is done as thoroughly as may be, a new record being secured for most cases every year. Fig. 8 shows the results obtained in the groups of cases fitted each year from 1951 to 1955; in each group the follow-up demonstrates the known wearing time in successive years up to September, 1955.

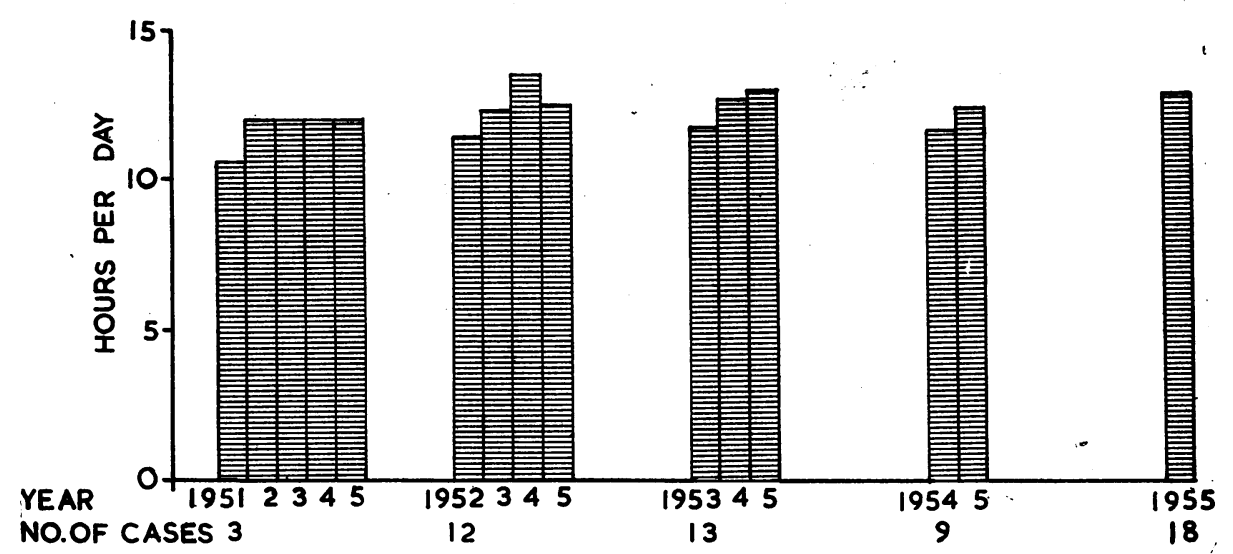

Fig. 8.-Average wearing-time. Cases grouped according to number of years for which lenses have been in use.

Summarizing this information about the 34 people who have had their lenses more than one year: we have lost trace of two, two are known to be abroad, and seven have given up-five on account of intercurrent disease or death, and two because they "can't be bothered". Our year-by-year records of the remaining 23 show that all still average 13 hours of wear daily.

This question of wearing contact lenses has been dealt with at some length because there is a persistent belief that, though these patients wear their lenses reasonably well at first they soon give them up. Our records prove that this is not true; our work is justified not only by the immediate restoration of useful sight to many young people otherwise doomed to near blindness in one or both eyes, but also by the acid test of long-term results.

\section{Summary}

The medical records of 92 cases of keratoconus sent to the Contact Lens Department between 1951 and 1955 are reviewed and the general picture of the disease as seen in this group of cases is set out. Particular attention is drawn to the importance of keeping a close watch on the fellow eye from the first moment a case of keratoconus is diagnosed. Any shift of the total 
refractive error in the second eye, usually towards myopia, is presumptive evidence that this eye is already affected by the disease. Since the majority of these cases become bilateral, and since the second eye usually becomes involved within 2 years of the first, this is a feasible clinical procedure which may assist in the discovery of the aetiology of the condition and enable treatment to be instituted much earlier. In only one case has evidence been obtained that an eye deteriorated after a contact lens was worn regularly, so that it seems possible that the wearing of contact lenses arrests the progress of the disease.

The 34 patients who have had their contact lenses for from 1 to 4 years were followed up. Only two had voluntarily given up wearing their lenses, and 23 who had worn them continuously and up to four years are known to average 13 hours of wear daily. It is concluded that contact lenses enable a high proportion of affected eyes to see usefully and even normally, that the lenses are tolerated well, and that the great improvement in vision as compared with that obtainable with spectacles is likely to be permanent.

Sincere thanks are due to all those surgeons who have entrusted their cases to us, to the Medical Officers of the department whose work is reported and interpreted in this paper, to the nonmedical staff, and in particular our Demonstrator-Fitter, Mr. T. C. Trodd, whose careful work has contributed so much to the results reported, to my secretary, Mrs White, for keeping the punch-card records, and much help in preparing the detailed analyses, and to Miss Ryder for her help in preparing the diagrams.

\section{REFERENCE}

RIDLEY, F. (1954). Trans. ophthal. Soc. U.K., 74, 377. 